\title{
Protective role and molecular mechanism of action of Nesfatin-1 against high glucose-induced inflammation, oxidative stress and apoptosis in retinal epithelial cells
}

\author{
HAIYAN SUN*, HUAHUI ZHAO*, ZHIPENG YAN, XIAOKUN LIU, \\ PENGFEI YIN and JUN ZHANG \\ Ophthalmology Department, The Third Hospital of Hebei Medical University, \\ Shijiazhuang, Hebei 050051, P.R. China
}

Received September 11, 2020; Accepted January 21, 2021

DOI: $10.3892 / \mathrm{etm} .2021 .10265$

\begin{abstract}
Diabetic retinopathy (DR) is a major complication of diabetes mellitus that may cause severe visual impairment. It has been reported that the levels of nesfatin-1 in the serum and vitreous humor were negatively correlated with DR; however, its role in DR has not been fully elucidated. Therefore, the present study was performed to investigate the effect of nesfatin-1 on high glucose-treated human retinal epithelial cells (ARPE-19) and explore the underlying mechanism. The effects of nesfatin-1 on cell viability, inflammation, oxidative stress and apoptosis were examined under high glucose conditions. The Cell Counting Kit-8 assay was used to determine cell viability. The levels of inflammatory cytokines were evaluated using ELISA kits. The reactive oxygen species and malondialdehyde content was estimated using commercial assay kits. Flow cytometry was performed to detect apoptotic cells and western blot analysis was employed to evaluate the expression of apoptosis-associated proteins. Moreover, the levels of NF- $\mathrm{BB}, \mathrm{NACHT}, \mathrm{LRR}$ and PYD domains-containing protein 3 (NLRP3) and high-mobility group protein B1 (HMGB1) were determined via western blot analysis. The results revealed that nesfatin-1 enhanced cell viability and suppressed inflammation, oxidative stress and apoptosis in the presence of high glucose concentration. Moreover, the activation of the NF- $\mathrm{kB} / \mathrm{NLRP} 3$ inflammasome signaling and the expression of HMGB1 were inhibited by nesfatin-1. Furthermore, HMGB1 overexpression partially abrogated
\end{abstract}

Correspondence to: Dr Jun Zhang, Ophthalmology Department, The Third Hospital of Hebei Medical University, 139 Ziqiang Road, Qiaoxi, Shijiazhuang, Hebei 050051, P.R. China

E-mail: zhangjunjz@yeah.net

"Contributed equally

Key words: high glucose, diabetic retinopathy, nesfatin-1, NACHT, LRR and PYD domains-containing protein 3, high-mobility group protein $\mathrm{B} 1$ the inactivation of the $\mathrm{NF}-\kappa \mathrm{B} / \mathrm{NLRP} 3$ inflammasome pathway caused by nesfatin- 1 . Taken together, these findings demonstrated that nesfatin-1 inhibited the activation of the $\mathrm{NF}-\kappa \mathrm{B} / \mathrm{NLRP} 3$ inflammasome signaling via modulating HMGB1 and exerted a protective effect on ARPE-19 cells against high glucose-induced inflammation, oxidative stress and apoptosis.

\section{Introduction}

Diabetes mellitus (DM) includes a group of metabolic conditions that are caused by disrupted carbohydrate metabolism due to islet dysfunction, which manifests as hyperglycemia (1). Chronic hyperglycemia results in the dysfunction of various tissues and organs, including the eyes, kidney, heart, vessels and nerves, with visual damage appearing in the early stages of DM (2). Diabetic retinopathy (DR) is a major classical microvascular complication of DM, resulting in retinal ischemia, altered retinal permeability, neovascularization and macular edema, eventually leading to visual impairment and blindness $(3,4)$. DR is classified into two stages: Non-proliferative diabetic retinopathy and proliferative diabetic retinopathy (PDR). PDR is the most advanced stage of the disease, and new vessels grow in a chaotic manner, causing vitreous hemorrhage and retinal detachment (5). Epidemiological data have revealed that 93 million individuals (35\% of diabetic adults aged 20-76 years) suffered from DR in 2010 worldwide, of whom 17 million exhibited PDR and 21 million displayed diabetic macular edema (3). It has been estimated that the number of DR cases will continue to increase by 2050 (6).

Vascular dysfunction, including endothelial cell damage, pericyte death, retinal capillary basement membrane thickening and tight junction alterations has been a major focus of DR research; however, diabetic vascular dysfunction alone cannot explain the loss of retinal function (7). Accumulating evidence has pointed to the key role of inflammation in the progression of DR (8-10). Previous studies have reported that the levels of various inflammatory cytokines, including IL-1 $\beta$, IL-6, IL-8, TNF- $\alpha$ and monocyte chemoattractant protein-1, were increased in the vitreous humor of patients with DR 
compared with healthy subjects (11-13). Of note, a recent study demonstrated that the serum and vitreous concentrations of nesfatin-1 were negatively correlated with DR (14).

Nesfatin-1 is a secretory peptide distributed in the hypothalamus and brainstem and is produced by the hydrolysis of nucleobindin-2 at its N-terminal (15). A previous study has suggested that nesfatin-1 may ameliorate the high glucose-induced toxicity in $\mathrm{PC} 12$ cells via the regulation of oxidative stress, autophagy and apoptosis (16). Furthermore, it has been demonstrated that nesfatin-1 suppressed NF- $\kappa \mathrm{B}$-dependent inflammatory responses and attenuated caspase-3-mediated neuronal cell apoptosis following traumatic brain injury in rats (17), indicating the anti-inflammatory and anti-apoptotic effects of nesfatin-1. However, the detailed function of nesfatin-1 in DR remains elusive.

High expression of NF- $\mathrm{KB}$ and NACHT, LRR and PYD domains-containing protein 3 (NLRP3) has been indicated to be closely associated with inflammation in DR (18-20). Enhanced expression level of NLRP3, caspase-1 and IL-1 $\beta$ has been observed in proliferative membranes of patients with DR compared with healthy subjects and in high glucose-treated human retinal endothelial cells compared with untreated cells, whereas inhibition of NLRP3 significantly alleviated inflammatory responses (21). In addition to NLRP3, high-mobility group protein B1 (HMGB1) has also been indicated to promote inflammation in DR, and inhibition of HMGB1 attenuated the NF- $\mathrm{\kappa B}$ activity in human retinal epithelial cells cultured with high glucose (22). Moreover, HMGB1 has been indicated to bind with the receptor for advanced glycation end products to accelerate rat retinal cell apoptosis (23). Previous studies have demonstrated that HMGB1 promoted the activation of the NLRP3 inflammasome, upregulated the expression level of NLRP3, apoptosis-associated speck-like protein containing a CARD (ASC) and caspase-1 $(24,25)$, whereas nesfatin-1 reduced the activation of the NF- $\mathrm{KB}$ pathway via downregulating the expression of HMGB1 (26). Therefore, the present study was performed to investigate the role of nesfatin- 1 in DR and explore the potential association among HMGB1, NF- $\kappa B$, NLRP3 and nesfatin-1.

\section{Materials and methods}

Cell culture and treatment. The human retinal epithelial cell line ARPE-19 was purchased from The Cell Bank of Type Culture Collection of Chinese Academy of Sciences and cultured in DMEM/F12 (HyClone; Cytiva) supplemented with $10 \%$ FBS (Gibco; Thermo Fisher Scientific, Inc.) at $37^{\circ} \mathrm{C}$ in an incubator with $95 \%$ air $/ 5 \% \mathrm{CO}_{2}$. In order to examine the effects of hyperglycemia, ARPE-19 cells were cultured with high concentration of glucose (33 mM; Sigma-Aldrich; Merck $\mathrm{KGaA})$, normal concentration of glucose $(5.5 \mathrm{mM})$ or $27.5 \mathrm{mM}$ mannitol $+5.5 \mathrm{mM}$ glucose as an osmotic control for $48 \mathrm{~h}$. ARPE-19 cells were pretreated with nesfatin-1 $(2.5$ or $5 \mathrm{ng} / \mathrm{ml}$; Sigma-Aldrich; Merck KGaA) for $1 \mathrm{~h}$, followed by exposure to $33 \mathrm{mM}$ glucose for $48 \mathrm{~h}(16)$.

Cell transfection. ARPE-19 cells $\left(2 \times 10^{5}\right.$ cells/well) were seeded into 6-well plates. Subsequently, cells were transfected with
HMGB1 plasmid (pcDNA3.1-HMGB1; $50 \mathrm{nM}$ ) or the empty vector plasmid (pcDNA3.1; $50 \mathrm{nM}$ ) using Lipofectamine ${ }^{\circledR} 3000$ (Invitrogen; Thermo Fisher Scientific, Inc.) at $37^{\circ} \mathrm{C}$, according to the manufacturer's instructions. These plasmids were provided by Shanghai GenePharma Co., Ltd. After 48 h, cells were treated with glucose/nesfatin as aforementioned. The transfected cells were harvested and protein expression was examined with western blot analysis.

Cell Counting Kit-8 (CCK-8) assay. Cell viability was determined using the CCK-8 assay. Briefly, ARPE-19 cells were seeded into a 96-well plate at a density of 5,000 cells/well. Following incubation with glucose and/or nesfatin- 1 as aforementioned, $10 \mu \mathrm{l}$ CCK- 8 solution (Nanjing KeyGen Biotech Co., Ltd.) was added into each well for $1 \mathrm{~h}$ at $37^{\circ} \mathrm{C}$. The absorbance of each well was measured using a microplate reader at $450 \mathrm{~nm}$.

Colony formation assay. ARPE-19 cells were seeded into 6-well plates (500 cells/well) and incubated for $24 \mathrm{~h}$ at $37^{\circ} \mathrm{C}$ to allow for adherence. The medium was changed every 3 days. After 2 weeks of culture, the cells were fixed with methanol for $30 \mathrm{~min}$ at room temperature and stained with $0.2 \%$ crystal violet at room temperature for $30 \mathrm{~min}$. Images were captured using a light microscope (Olympus Corporation) at x10 magnification.

ELISA. ARPE-19 cell culture medium was collected into a centrifuge tube and centrifuged at $1,000 \mathrm{x} \mathrm{g}$ for $10 \mathrm{~min}$ at $4^{\circ} \mathrm{C}$. Subsequently, the concentration of TNF- $\alpha$ (cat. no. SEKH-0047; Beijing Solarbio Science \& Technology Co., Ltd.), IL-1 $\beta$ (cat. no. F01220) and IL-6 (cat. no. F01310; both from Shanghai Xitang Biotechnology Co., Ltd.) was measured by ELISA kits according to the manufacturer's instructions. The absorbance of each well was measured using a microplate reader at $450 \mathrm{~nm}$. The concentration of nesfatin-1 was determined with a commercially available ELISA kit (cat. no. JL19919-96T; Shanghai Jianglai Biological Technology Co., Ltd.) in accordance with the manufacturer's instructions.

Measurement of oxidative stress-related malondialdehyde (MDA). ARPE-19 cells were collected into a centrifuge tube and centrifuged at $8,000 \mathrm{xg}$ for $10 \mathrm{~min}$ at $4^{\circ} \mathrm{C}$. Subsequently, the reagents of the MDA assay kit (cat. no. BC0025; Beijing Solarbio Science \& Technology Co., Ltd.) were added into the centrifuge tube according to the manufacturer's protocol. The mixture was heated at $100^{\circ} \mathrm{C}$ for $60 \mathrm{~min}$, cooled on ice and centrifuged at $10,000 \mathrm{xg}$ for $10 \mathrm{~min}$ at room temperature. Subsequently, $200 \mu 1$ supernatant was added into a 96-well plate. The absorbance of each well was recorded using a microplate reader at $532 \mathrm{~nm}$.

Measurement of reactive oxygen species (ROS). The production of intracellular ROS was determined with a ROS assay kit (cat. no. D6470; Beijing Solarbio Science \& Technology Co.,Ltd.) using 2',7'-dichlorodihydrofluorescein diacetate (DCFH-DA) as a fluorescence probe. ARPE-19 cells were seeded into a 6-well plate, and DCFH-DA $(10 \mu \mathrm{M})$ was added into each well according to the manufacturer's protocol for $20 \mathrm{~min}$ at $37^{\circ} \mathrm{C}$. 

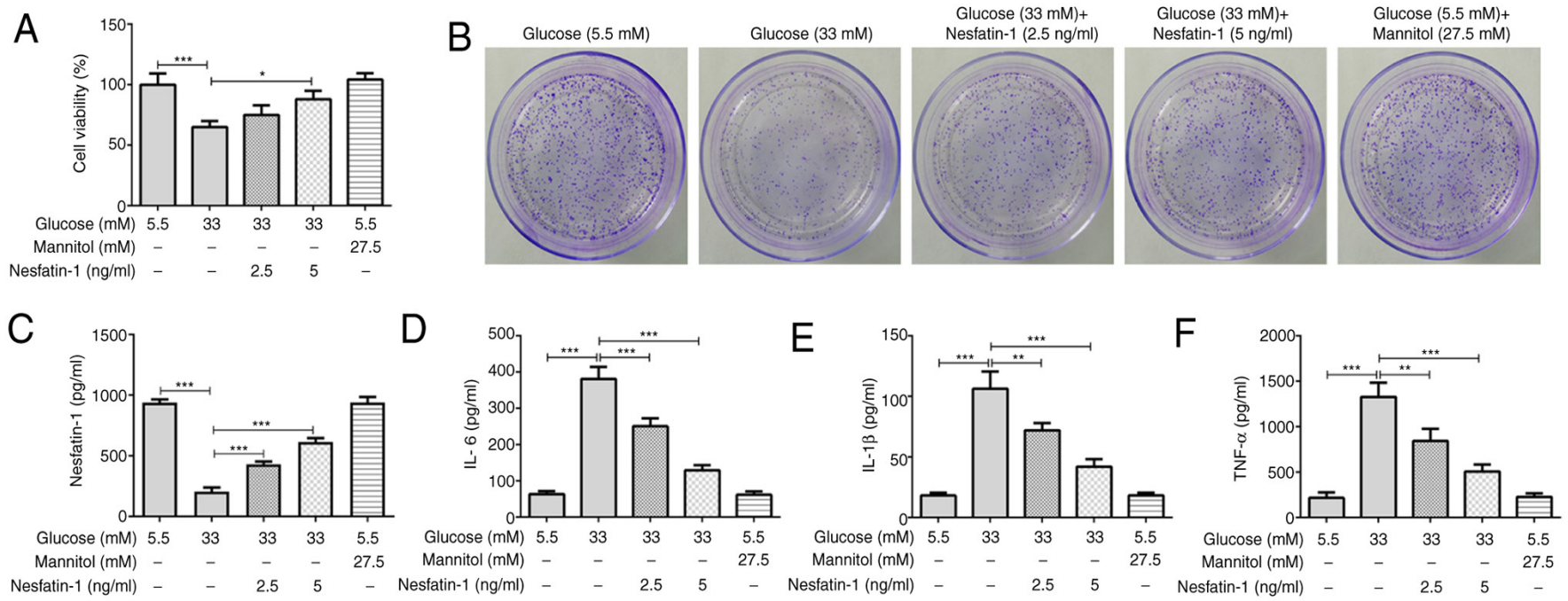

Figure 1. Nesfatin-1 enhances cell viability and decreases inflammatory responses in high glucose-treated ARPE-19 cells. (A) Cell viability in each group was assessed using Cell Counting Kit- 8 assay. (B) Cell proliferative ability was evaluated with colony formation assay. (C) The levels of nesfatin-1 were determined using ELISA. (D-F) The concentration of the indicated pro-inflammatory cytokines in the cell culture medium of each group was determined using ELISA. ${ }^{*} \mathrm{P}<0.05 ;{ }^{* *} \mathrm{P}<0.01 ;{ }^{* * * *} \mathrm{P}<0.001$.

Subsequently, the cells were washed with DMEM/F12 three times and the absorbance of each well was measured using a fluorescence microplate reader at 488 and $525 \mathrm{~nm}$.

Flow cytometry analysis. To determine apoptosis, $2 \times 10^{5}$ ARPE-19 cells were prepared and washed with PBS twice. Subsequently, the cells were suspended in $500 \mu 1$ binding buffer (Nanjing KeyGen Biotech Co., Ltd.), followed by the addition of $5 \mu \mathrm{l}$ Annexin V-FITC and $5 \mu \mathrm{l}$ PI (Nanjing KeyGen Biotech Co., Ltd.) for $10 \mathrm{~min}$ at room temperature in the dark. Subsequently, cell apoptosis was analyzed using a flow cytometer (FACSAria III; BD Biosciences). The data were analyzed using BD Accuri C6 software (version 1.0; Becton, Dickinson and Company).

Western blot analysis. Total protein from ARPE-19 cells was extracted using a RIPA lysis buffer (Beyotime Institute of Biotechnology). Then, ARPE-19 cells were collected into a centrifuge tube and centrifuged at $10,000 \mathrm{x}$ g for $10 \mathrm{~min}$ at $4^{\circ} \mathrm{C}$. The cell lysate was collected into another centrifuge tube and the protein concentration of the lysate was determined using a BCA assay kit (Sigma-Aldrich; Merck KGaA). A total of $20 \mu \mathrm{g}$ protein lysate was loaded to $10 \%$ SDS-PAGE gels and then transferred to PVDF membranes (EMD Millipore). The membranes were incubated with primary antibodies at $4^{\circ} \mathrm{C}$ overnight after blocking with $5 \%$ non-fat milk for $1 \mathrm{~h}$ at room temperature. Primary antibodies against NF- $\kappa$ B (cat. no. ab32536; 1:1,000), NLRP3 (cat. no. ab263899; 1:1,000), caspase-1 (cat. no. ab179515; 1:1,000), pro-caspase-1 (cat. no. ab207802; 1:1,000), ASC (cat. no. ab151700; 1:1,000), HMGB1 (cat. no. ab18256; 1:1,000), Bcl-2 (cat. no. ab32124; 1:1,000), Bax (cat. no. ab32503; 1:1,000) and GAPDH (cat. no. ab8245; 1:1,000) were all obtained from Abcam. Following incubation with HRP-conjugated goat anti-rabbit IgG (cat. no. ab205718; Abcam; 1:5,000) or goat anti-mouse IgG (cat. no. ab6789; Abcam; 1:5,000) secondary antibodies at $37^{\circ} \mathrm{C}$ for $1 \mathrm{~h}$, the protein bands of the membranes were detected using Immobilon Western Chemiluminescent
HRP Substrate (EMD Millipore). The relative intensity of target bands was semi-quantified using Image J software (version 1.52r; National Institutes of Health) and normalized to the intensity of GAPDH.

Statistical analysis. Data are presented as mean \pm SD. All experiments were performed in triplicate. Statistical analyses were carried out using GraphPad Prism v6 software (GraphPad Software, Inc.). One-way ANOVA followed by Tukey's post hoc test was used to compare the differences among groups. $\mathrm{P}<0.05$ was considered to indicate a statistically significant difference.

\section{Results}

Nesfatin-1 enhances cell viability and decreases inflammatory responses in high glucose-treated ARPE-19 cells. ARPE-19 cells exposed to high glucose exhibited lower viability compared with that of the normal glucose group. Nesfatin-1 treatment alleviated the decrease in cell viability induced by high glucose, which was dose-dependent. The osmotic group revealed that osmotic pressure exerted no significant effects on cell viability, excluding the effect of osmotic pressure on the high glucose group (Fig. 1A). The results of the colony formation assay indicated that high glucose exposure inhibited the proliferation of ARPE-19 cells compared with the normal glucose group, while nesfatin-1 treatment remarkably increased the cell proliferation relatively to the high glucose group (Fig. 1B). Additionally, a notably reduced nesfatin-1 level was observed following high glucose stimulation, whereas nesfatin-1 treatment significantly enhanced the nesfatin-1 level (Fig. 1C). Moreover, osmotic pressure presented no effect on the levels of IL-6, IL-1 $\beta$ and TNF- $\alpha$ compared with the normal glucose group, but high glucose significantly induced the secretion of these inflammatory cytokines, whereas nesfatin-1 treatment reduced the levels of inflammatory cytokines compared with the high glucose group (Fig. 1D-F). 
A

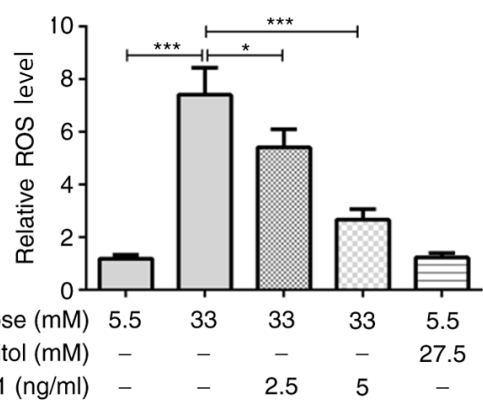

B

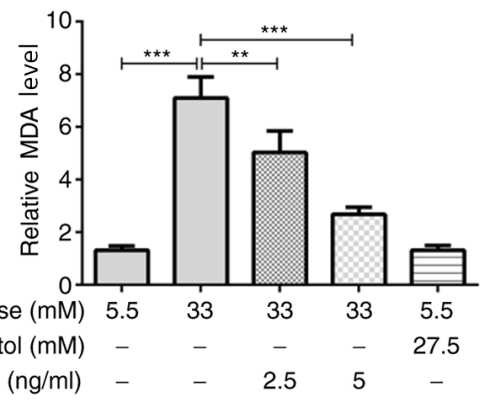

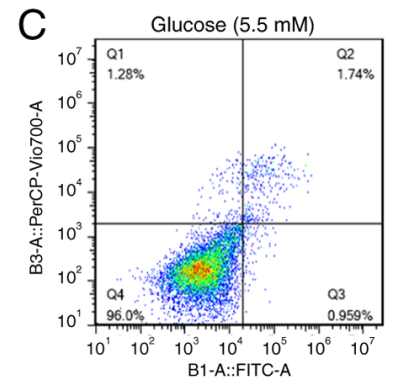

Glucose $(33 \mathrm{mM})+$ Nesfatin-1

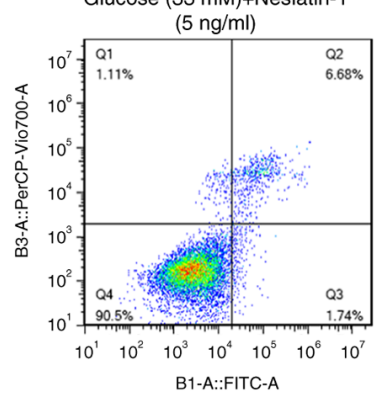

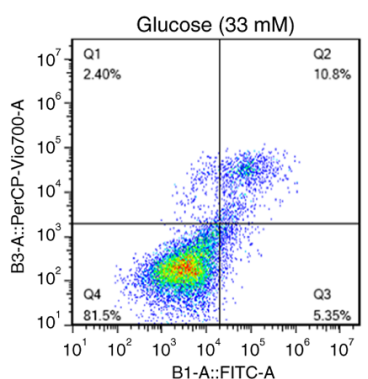

Glucose $(5.5 \mathrm{mM})+$ Mannitol

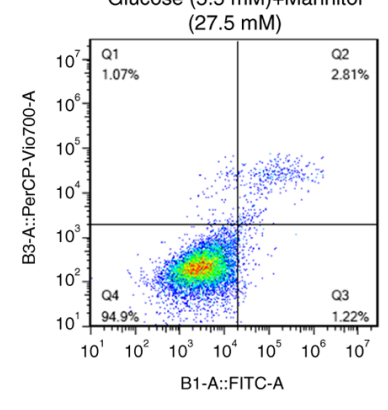

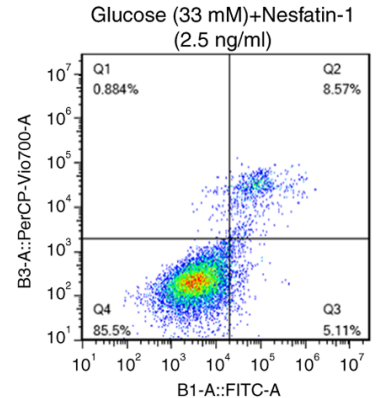

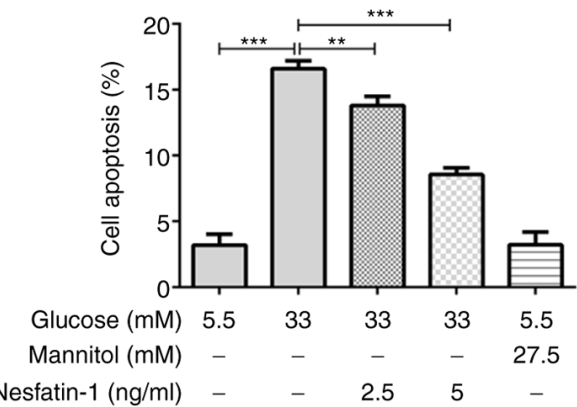

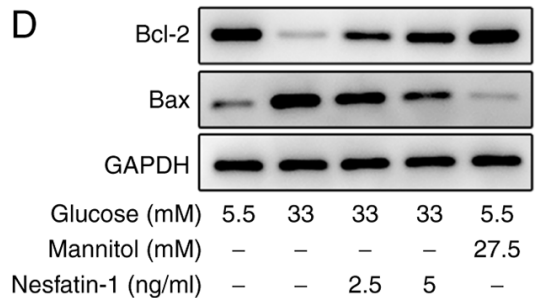

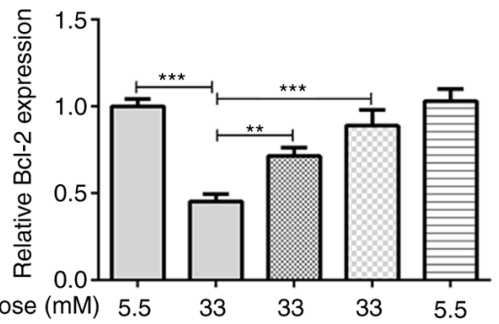
$\begin{array}{cccccc}\text { Glucose }(\mathrm{mM}) & 5.5 & 33 & 33 & 33 & 5.5 \\ \text { Mannitol }(\mathrm{mM}) & - & - & - & - & 27.5\end{array}$ Nesfatin-1 (ng/ml)

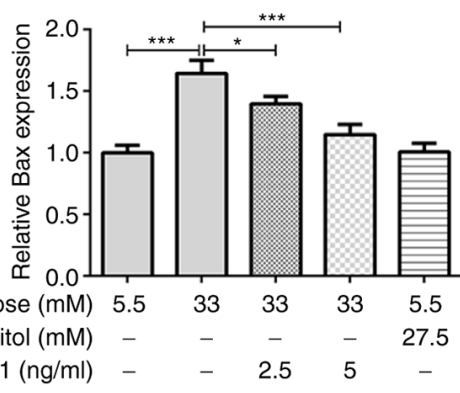

Figure 2. Nesfatin-1 alleviates high glucose-induced oxidative stress and apoptosis in ARPE-19 cells. (A) The ROS and (B) MDA content in each group was determined with commercial assay kits. (C) The apoptotic rate was assessed via flow cytometry. (D) The level of the pro-apoptotic protein Bax and the anti-apoptotic protein Bcl-2 was examined via western blot analysis. ${ }^{*} \mathrm{P}<0.05 ;{ }^{* *} \mathrm{P}<0.01 ;{ }^{* * * *} \mathrm{P}<0.001$. ROS, reactive oxygen species; MDA, malondialdehyde.

Nesfatin-1 alleviates high glucose-induced oxidative stress and apoptosis in ARPE-19 cells. A number of studies have demonstrated that high glucose concentration can induce cellular oxidative stress $(27,28)$. It was observed that osmotic pressure exhibited no effect on the levels of ROS and MDA and high glucose markedly increased the levels of ROS and MDA compared with the normal glucose group, whereas nesfatin-1 reduced the cellular ROS and MDA content. Furthermore, the high dose of nesfatin-1 exerted a more potent antioxidant effect compared with the low dose nesfatin-1 (Fig. 2A and B). Consistently, there was no significant difference in cell apoptosis between the mannitol group and the normal glucose group, but nesfatin-1 reduced high glucose-induced cell apoptosis (Fig. 2C). In addition, osmotic pressure exhibited no effect on the expression of Bcl-2 and Bax compared with the normal glucose group. However, the decreased Bcl-2 and increased Bax level in high glucose-treated ARPE-19 cells also demonstrated that high glucose concentration may increase the cell apoptosis rate, whereas nesfatin-1 treatment reversed the effect of high glucose on the protein level of Bcl-2 and Bax (Fig. 2D). These data provided evidence that nesfatin-1 attenuated high glucose-induced oxidative stress and apoptosis in ARPE-19 cells. 
A
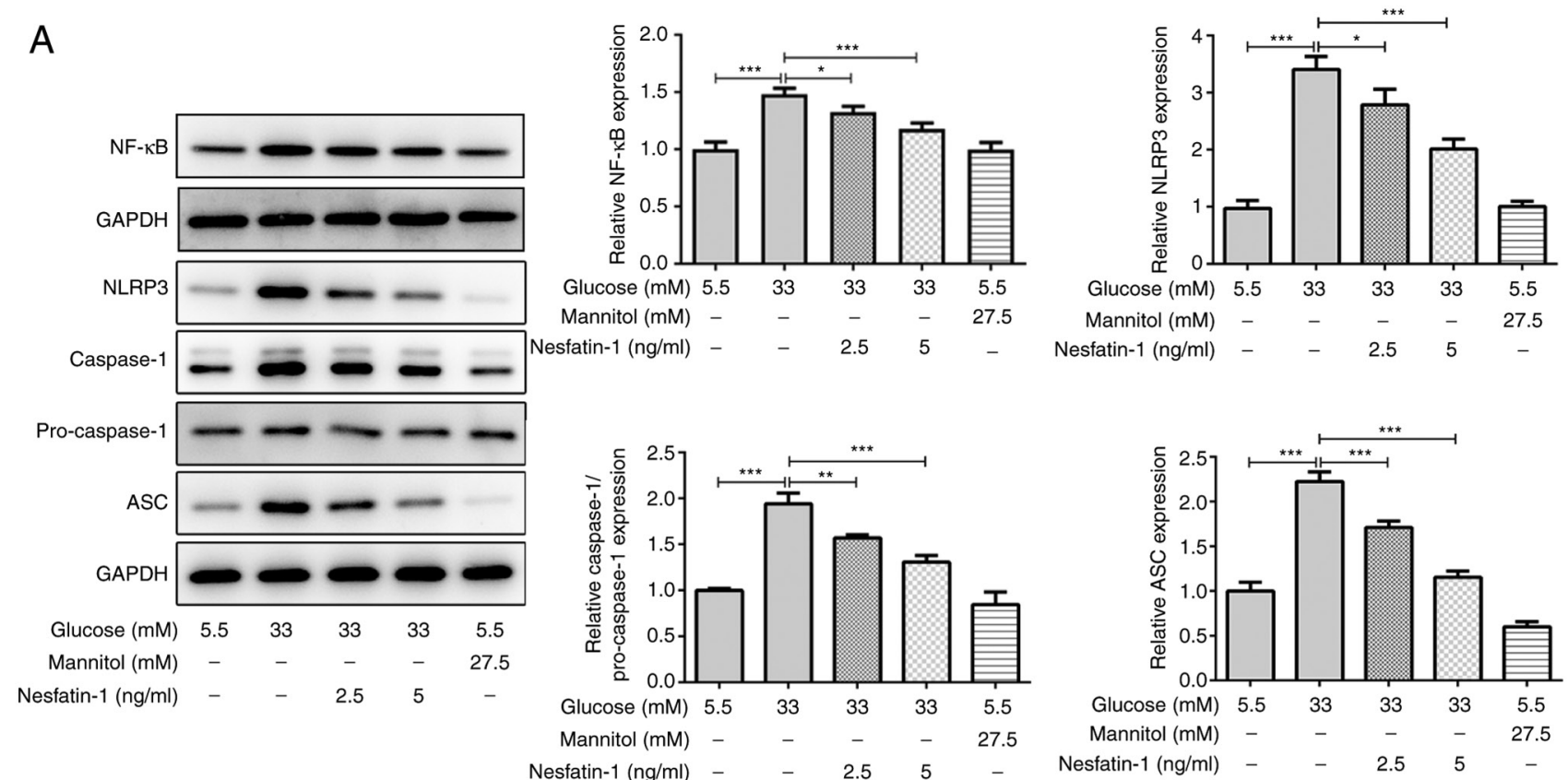

B
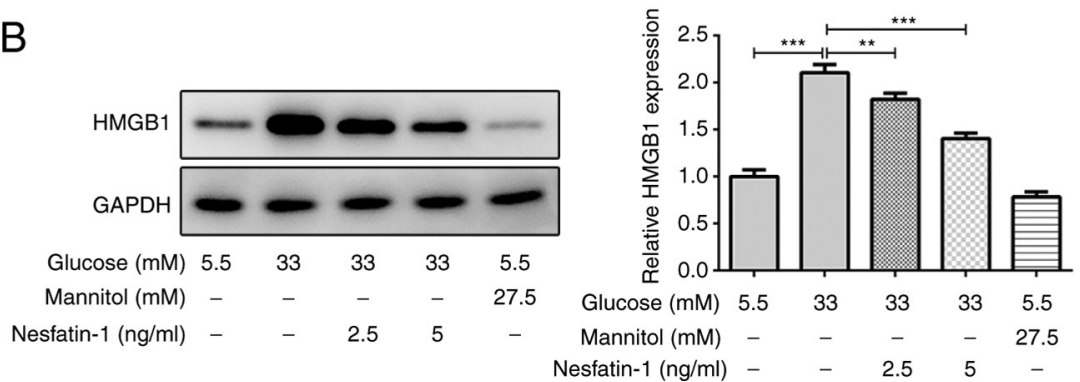

Figure 3. Nesfatin-1 inhibits the activation of NF- $\mathrm{BB} / \mathrm{NLRP} 3$ inflammasome signaling and HMGB1 expression in ARPE-19 cells stimulated by high glucose. (A) The protein expression level of NF-kB and NLRP3 inflammasome proteins, including NLRP3, ASC and caspase-1, and (B) the protein expression level of HMGB1 in each group was determined using western blot analysis. ${ }^{*} \mathrm{P}<0.05 ;{ }^{* *} \mathrm{P}<0.01 ;{ }^{* * *} \mathrm{P}<0.001$. NLRP3, NACHT, LRR and PYD domains-containing protein 3; ASC, apoptosis-associated speck-like protein containing a CARD; HMGB1, high-mobility group protein B1.

Nesfatin-1 inhibits the activation of $N F-\kappa B / N L R P 3$ inflammasome signaling and $H M G B 1$ expression in high glucose-stimulated ARPE-19 cells. High glucose concentration stimulates the activation of the NF- $\mathrm{KB} / \mathrm{NLRP} 3$ inflammasome pathway, which has been indicated to be associated with the dysfunction of high glucose-treated human retinal endothelial cells $(21,29)$. There was no significant difference in the expression of NF- $\mathrm{KB}$, NLRP3, caspase-1 and ASC between the mannitol group and the normal glucose group. In high glucose-stimulated ARPE-19 cells, the protein expression levels of NF-KB, NLRP3, caspase-1 and ASC were increased, whereas the levels of these proteins were markedly reduced by nesfatin-1 treatment (Fig. 3A), indicating that nesfatin-1 inhibited the NF- $\kappa$ B/NLRP3 inflammasome signaling activation induced by high glucose exposure. Furthermore, osmotic pressure exhibited no significant effect on the expression of HMGB1, but the expression level of HMGB1 was markedly increased in high glucose-treated ARPE-19 cells, and it was reduced following nesfatin-1 treatment (Fig. 3B). Overall, these data suggested that nesfatin-1 suppressed the NF- $\mathrm{kB} / \mathrm{NLRP} 3$ inflammasome pathway activation and HMGB1 expression in high glucose-stimulated ARPE-19 cells.
Nesfatin-1 prevents the activation of $N F-\kappa B / N L R P 3$ inflammasome signaling via regulating $H M G B 1$. Subsequently, the mechanism via which nesfatin-1 affected the activation of the NF- $\kappa$ B/NLRP3 inflammasome pathway was further investigated. ARPE-19 cells were transfected with pcDNA3.1-HMGB1. As demonstrated in Fig. 4A, pcDNA3.1-HMGB1 increased the expression level of HMGB1 compared with the empty vector, suggesting the efficiency of pcDNA3.1-HMGB1 transfection. Of note, under high glucose conditions, nesfatin-1 inhibited NF- $\mathrm{kB} / \mathrm{NLRP} 3$ inflammasome signaling, and this effect could be reversed via upregulation of HMGB1 (Fig. 4B), indicating that nesfatin-1 prevented the activation of NF- $\mathrm{KB} / \mathrm{NLRP} 3$ inflammasome signaling via the regulation of HMGB1.

\section{Discussion}

Nesfatin-1 is considered to have various functions in different systems and metabolic processes, including the endocrine and nervous system, blood glucose concentration, cardiovascular system and lipid metabolism $(30,31)$. It has been reported that the concentration of nesfatin-1 in the serum and vitreous humor was negatively correlated with DR (14). However, the 
A
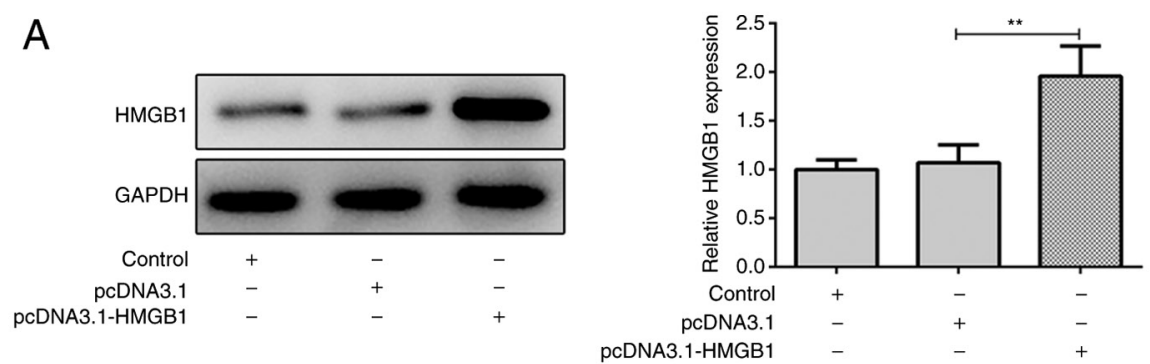

B
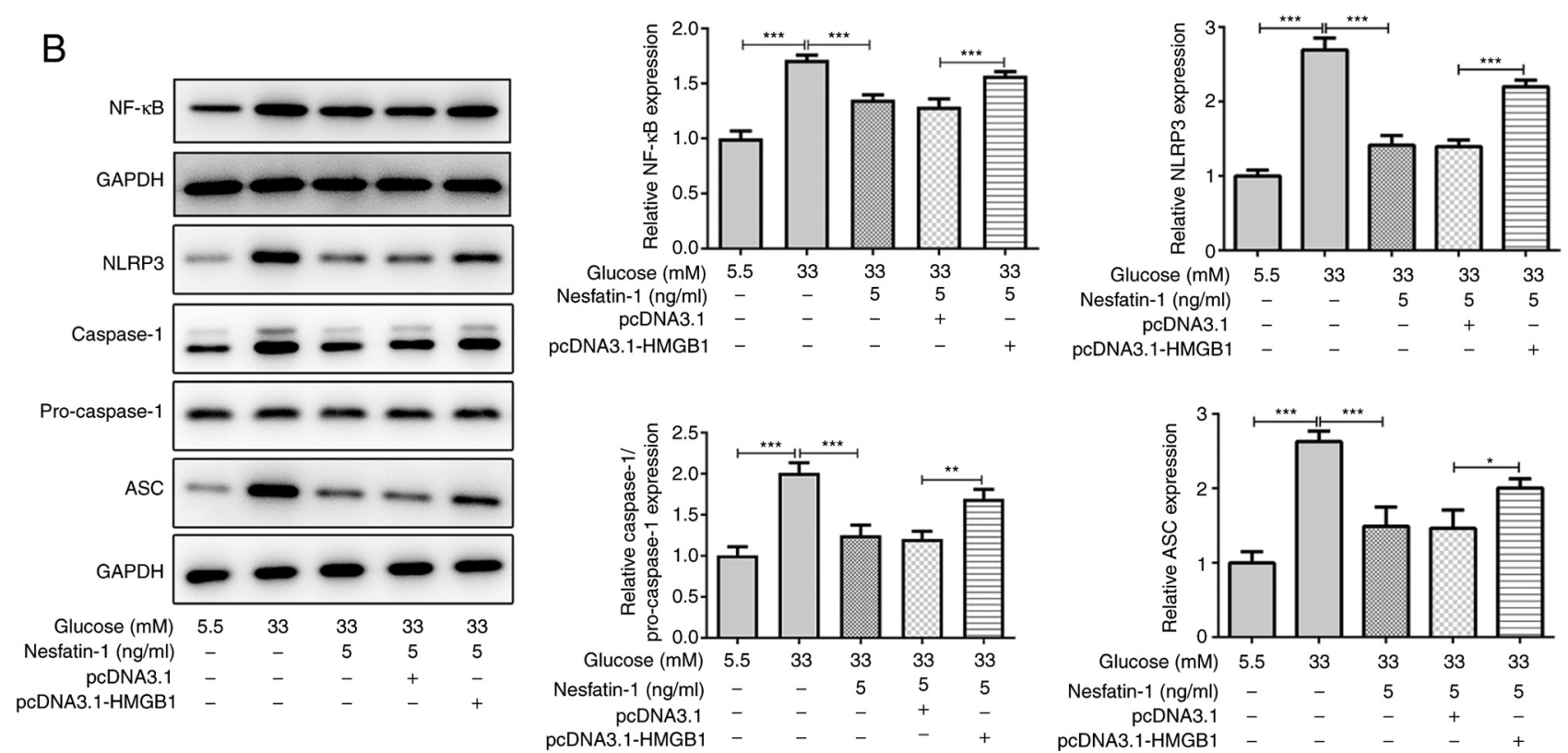

Figure 4. Nesfatin-1 prevents the activation of NF- $\mathrm{KB} / \mathrm{NLRP} 3$ inflammasome signaling via regulating HMGB1 in high glucose-treated ARPE-19 cells. (A) The transfection efficiency of HMGB1 overexpression plasmid was validated via western blot analysis. (B) The protein expression level of NF-кB and NLRP3 inflammasome proteins, including NLRP3, ASC and caspase-1, was evaluated using western blot analysis. ${ }^{*} \mathrm{P}<0.05 ;{ }^{* * *} \mathrm{P}<0.01 ;{ }^{* * * *} \mathrm{P}<0.001$. NLRP3, NACHT, LRR and PYD domains-containing protein 3; ASC, apoptosis-associated speck-like protein containing a CARD; HMGB1, high-mobility group protein B1.

role of nesfatin-1 in DR has not been extensively investigated to date.

Retinal pigment epithelial cells are the main cells involved in DR (32). In the present study, ARPE-19 human retinal epithelial cells were cultured with high glucose in vitro to mimic hyperglycemia in vivo. It has been previously reported that ROS-induced oxidative stress and low-grade inflammation triggered by chronic hyperglycemia contribute to the progression of DR (33). In the current study, it was observed that high glucose concentration reduced cell viability, induced the expression of inflammatory cytokines and increased the ROS and MDA content following high glucose stimulation. Nesfatin-1 treatment enhanced cell viability and suppressed the levels of TNF- $\alpha$, IL-1 $\beta$, IL- 6 , ROS and MDA in high glucose-treated cells, suggesting that nesfatin-1 may protect ARPE-19 cells against high glucose-induced inflammation and oxidative stress. In addition, nesfatin-1 decreased cell apoptosis under high glucose conditions.

NLRP3 inflammasome is a protein complex in the innate immune system that recognizes pathogen- and danger-associated molecular patterns, which is composed of NLRP3, ASC and caspase-1 (34). Activation of the NF- $\kappa \mathrm{B} / \mathrm{NLRP} 3$ inflammasome signaling serves a key role in the progression of DR (19,20,35-38). In the present study, it was observed that nesfatin-1 reduced the protein levels of NF- $\kappa \mathrm{B}, \mathrm{NLRP} 3, \mathrm{ASC}$ and caspase-1, and inhibited the activation of the NF- $\mathrm{B}$ /NLRP3 inflammasome signaling following high glucose stimulation. HMGB1 is a danger-associated molecular pattern receptor that can sense high glucose as a stressor (39). Elevated HMGB1 levels were observed in patients with advanced DR compared with healthy subjects (39). A recent study reported that HMGB1 was revealed to be highly expressed in high glucose-treated human retinal endothelial cells, and it may be associated with the pathogenesis of DR (40). Emerging evidence supports that nesfatin-1 can downregulate the expression of HMGB1 to alleviate lipopolysaccharide-induced acute lung injury (26). The present study demonstrated that high glucose concentration increased the expression level of HMGB1 in ARPE-19 cells, which was reduced by nesfatin-1 treatment. Of note, overexpression of HMGB1 partially reversed the inhibitory effect of nesfatin-1 on $\mathrm{NF}-\kappa \mathrm{B} / \mathrm{NLRP} 3$ inflammasome signaling activation, suggesting that nesfatin-1 may prevent the NF- $\mathrm{B} / \mathrm{NLRP} 3$ inflammasome pathway activation via inhibiting HMGB1. Consistently, previous studies have reported that HMGB1 increased the expression level of NF- $\kappa \mathrm{B}$ and NLRP3 inflammasome proteins, including NLRP3, ASC, caspase-1 or IL-1 $\beta(25,41,42)$.

In summary, the present study demonstrated the beneficial effect of nesfatin-1 in high glucose-treated ARPE-19 cells via 
inhibiting inflammation, oxidative stress and apoptosis. To the best of our knowledge, the present study was the first to demonstrate that nesfatin-1 prevented the NF- $\mathrm{B} / \mathrm{NLRP} 3$ inflammasome signaling activation via the inhibition of HMGB1. The role of nesfatin-1 in animal experiments, the mechanisms mediating the reduction of HMGB1 by nesfatin-1 in and the effective dose of nesfatin-1 for clinical applications, which are limitations to the present study, should be investigated in future studies.

\section{Acknowledgements}

Not applicable.

\section{Funding}

No funding was received.

\section{Availability of materials and data}

The datasets used and/or analyzed during the current study are available from the corresponding author on reasonable request.

\section{Authors' contributions}

HS, HZ and ZY searched the literature, designed and performed the experiments. XL and PY analyzed the data and wrote the manuscript. JZ analyzed the data and revised the manuscript. HS and JZ confirm the authenticity of the raw data. All authors read and approved the final manuscript.

\section{Ethics approval and consent to participate}

Not applicable.

\section{Patient consent for publication}

Not applicable.

\section{Competing interests}

The authors declare that they have no competing interests.

\section{References}

1. Toniolo A, Cassani G, Puggioni A, Rossi A, Colombo A Onodera $\mathrm{T}$ and Ferrannini E: The diabetes pandemic and associated infections: Suggestions for clinical microbiology. Rev Med Microbiol 30: 1-17, 2019.

2. Vieira-Potter VJ, Karamichos D and Lee DJ: Ocular complications of diabetes and therapeutic approaches. Biomed Res Int 2016: 3801570, 2016.

3. Yau JW, Rogers SL, Kawasaki R, Lamoureux EL, Kowalski JW, Bek T, Chen SJ, Dekker JM, Fletcher A, Grauslund J, et al: Global prevalence and major risk factors of diabetic retinopathy. Diabetes Care 35: 556-564, 2012.

4. Duh EJ, Sun JK and Stitt AW: Diabetic retinopathy: Current understanding, mechanisms, and treatment strategies. JCI insight 2: e93751, 2017.

5. Ahmadieh H, Behbahani S and Safi S: Continuous wavelet transform analysis of ERG in patients with diabetic retinopathy. Doc Ophthalmol 142: 305-314, 2021.

6. Saaddine JB, Honeycutt AA, Narayan KM, Zhang X, Klein R and Boyle JP: Projection of diabetic retinopathy and other major eye diseases among people with diabetes mellitus: United States, 2005-2050. Arch Ophthalmol 126: 1740-1747, 2008.
7. Rübsam A, Parikh S and Fort PE: Role of inflammation in diabetic retinopathy. Int J Mol Sci 19: 942, 2018.

8. Noda K, Nakao S, Ishida S and Ishibashi T: Leukocyte adhesion molecules in diabetic retinopathy. J Ophthalmol 2012: 279037, 2012.

9. Kern TS: Contributions of inflammatory processes to the development of the early stages of diabetic retinopathy. Exp Diabetes Res 2007: 95103, 2007.

10. Adamis AP: Is diabetic retinopathy an inflammatory disease? Br J Ophthalmol 86: 363-365, 2002.

11. Maier R, Weger M, Haller-Schober EM, El-Shabrawi Y, Wedrich A, Theisl A, Aigner R, Barth A and Haas A: Multiplex bead analysis of vitreous and serum concentrations of inflammatory and proangiogenic factors in diabetic patients. Mol Vis 14: 637-643, 2008

12. Dai Y, Wu Z, Wang F, Zhang Z and Yu M: Identification of chemokines and growth factors in proliferative diabetic retinopathy vitreous. BioMed Res Int 2014: 486386, 2014.

13. Yoshimura T, Sonoda KH, Sugahara M, Mochizuki Y, Enaida H, Oshima Y, Ueno A, Hata Y, Yoshida H and Ishibashi T: Comprehensive analysis of inflammatory immune mediators in vitreoretinal diseases. PLoS One 4: e8158, 2009.

14. Dai R, Deng G, Sun Z, Liu Z, Qian Y and Han Y: Relation of serum and vitreous nesfatin-1 concentrations with diabetic retinopathy. J Clin Lab Anal 31: e22105, 2017.

15. Shimizu H, Oh IS, Hashimoto K, Nakata M, Yamamoto S, Yoshida N, Eguchi H, Kato I, Inoue K, Satoh T, et al: Peripheral administration of nesfatin-1 reduces food intake in mice: The leptin-independent mechanism. Endocrinology 150: 662-671, 2009.

16. Nazarnezhad S, Rahmati M, Shayannia A, Abbasi Z, Salehi M and Khaksari M: Nesfatin-1 protects PC12 cells against high glucose-induced cytotoxicity via inhibiting oxidative stress, autophagy and apoptosis. Neurotoxicology 74: 196-202, 2019.

17. Tang $\mathrm{CH}, \mathrm{Fu} \mathrm{XJ}, \mathrm{Xu} \mathrm{XL}$, Wei $\mathrm{XJ}$ and Pan HS: The anti-inflammatory and anti-apoptotic effects of nesfatin- 1 in the traumatic rat brain. Peptides 36: 39-45, 2012.

18. Chen H, Zhang X, Liao N, Mi L, Peng Y, Liu B, Zhang S and Wen F: Enhanced expression of NLRP3 inflammasome-related inflammation in diabetic retinopathy. Invest Ophthalmol Vis Sci 59: 978-985, 2018.

19. Yin Y, Chen F, Wang W, Wang H and Zhang X: Resolvin D1 inhibits inflammatory response in STZ-induced diabetic retinopathy rats: Possible involvement of NLRP3 inflammasome and NF- $\kappa$ B signaling pathway. Mol Vis 23: 242-250, 2017.

20. Yang Q, Li S, Zhou Z, Fu M, Yang X, Hao K and Liu Y: HDAC6 inhibitor Cay10603 inhibits high glucose-induced oxidative stress, inflammation and apoptosis in retinal pigment epithelial cells via regulating NF- $\kappa \mathrm{B}$ and NLRP3 inflammasome pathway. Gen Physiol Biophys 39: 169-177, 2020.

21. Zhang Y, Lv X, Hu Z, Ye X, Zheng X, Ding Y, Xie P and Liu Q: Protection of Mcc950 against high-glucose-induced human retinal endothelial cell dysfunction. Cell Death Dis 8: e2941, 2017.

22. Chen XL, Zhang XD, Li YY, Chen XM, Tang DR and Ran RJ: Involvement of HMGB1 mediated signalling pathway in diabetic retinopathy: Evidence from type 2 diabetic rats and ARPE-19 cells under diabetic condition. Br J Ophthalmol 97: 1598-1603, 2013.

23. Yu Y, Yang L, Lv J, Huang X, Yi J, Pei C and Shao Y: The role of high mobility group box 1 (HMGB-1) in the diabetic retinopathy inflammation and apoptosis. Int J Clin Exp Pathol 8: 6807-6813, 2015.

24. Chi W, Chen H, Li F, Zhu Y, Yin W and Zhuo Y: HMGB1 promotes the activation of NLRP3 and caspase- 8 inflammasomes via NF- $\mathrm{B}$ pathway in acute glaucoma. J Neuroinflammation 12: 137, 2015.

25. Kim EJ, Park SY, Baek SE, Jang MA, Lee WS, Bae SS, Kim K and Kim CD: HMGB1 Increases IL-1 $\beta$ production in vascular smooth muscle cells via NLRP3 Inflammasome. Front Physiol 9: 313, 2018.

26. Wang ZZ, Chen SC, Zou XB, Tian LL, Sui SH and Liu NZ: Nesfatin-1 alleviates acute lung injury through reducing inflammation and oxidative stress via the regulation of HMGB1. Eur Rev Med Pharmacol Sci 24: 5071-5081, 2020.

27. Chen X, Shen WB, Yang P, Dong D, Sun W and Yang P: High glucose inhibits neural stem cell differentiation through oxidative stress and endoplasmic reticulum stress. Stem Cells Dev 27: 745-755, 2018. 
28. Farnoodian M, Halbach C, Slinger C, Pattnaik BR, Sorenson CM and Sheibani N: High glucose promotes the migration of retinal pigment epithelial cells through increased oxidative stress and PEDF expression. Am J Physiol Cell Physiol 311: C418-C436, 2016.

29. Hu YJ, Lin HJ, Dib B, Atik A, Bouzika P, Lin C, Yan Y, Tang S, Miller JW and Vavvas DG: Cholesterol crystals induce inflammatory cytokines expression in a human retinal pigment epithelium cell line by activating the NF- $\kappa \mathrm{B}$ pathway. Discov Med 18: 7-14, 2014.

30. Tekin T, Cicek B and Konyaligil N: Regulatory peptide nesfatin-1 and its relationship with metabolic syndrome. Eurasian J Med 51: 280-284, 2019.

31. Ayada C, Toru Ü and Korkut Y: Nesfatin-1 and its effects on different systems. Hippokratia 19: 4-10, 2015.

32. Shivarudrappa AH and Ponesakki G: Lutein reverses hyperglycemia-mediated blockage of $\mathrm{Nrf} 2$ translocation by modulating the activation of intracellular protein kinases in retinal pigment epithelial (ARPE-19) cells. J Cell Commun Signal 14: 207-221, 2020

33. Singh LP: Thioredoxin interacting protein (TXNIP) and pathogenesis of diabetic retinopathy. J Clin Exp Ophthalmol 4: 287, 2013.

34. Ratsimandresy RA, Dorfleutner A and Stehlik C: An update on PYRIN domain-containing pattern recognition receptors: From immunity to pathology. Front Immunol 4: 440, 2013.

35. Li W, Liu X, Tu Y, Ding D, Yi Q, Sun X, Wang Y, Wang K, Zhu M and Mao J: Dysfunctional Nurrl promotes high glucose-induced Müller cell activation by up-regulating the NF- $\kappa \mathrm{B} / \mathrm{NLRP} 3$ inflammasome axis. Neuropeptides 82: 102057, 2020.
36. Lu L, Lu Q, Chen W, Li J, Li C and Zheng Z: Vitamin $\mathrm{D}_{3}$ protects against diabetic retinopathy by inhibiting high-glucose-induced activation of the ROS/TXNIP/NLRP3 inflammasome pathway. J Diabetes Res 2018: 8193523, 2018.

37. Li S, Yang H and Chen X: Protective effects of sulforaphane on diabetic retinopathy: Activation of the Nrf2 pathway and inhibition of NLRP3 inflammasome formation. Exp Animals 68: 221-231, 2019.

38. Loukovaara S, Piippo N, Kinnunen K, Hytti M, Kaarniranta K and Kauppinen A: NLRP3 inflammasome activation is associated with proliferative diabetic retinopathy. Acta Ophthalmol 95: 803-808, 2017.

39. Steinle JJ: Role of HMGB1 signaling in the inflammatory process in diabetic retinopathy. Cell Signal 73: 4, 2020.

40. Liang WJ, Yang HW, Liu HN, Qian W and Chen XL: HMGB1 upregulates NF-kB by inhibiting IKB- $\alpha$ and associates with diabetic retinopathy. Life Sci 241: 117146, 2020.

41. Duan J,Zhang Q, Hu X, Lu D, Yu W and Bai H: $\mathrm{N}^{4}$-acetylcytidine is required for sustained NLRP3 inflammasome activation via HMGB1 pathway in microglia. Cell Signal 58: 44-52, 2019.

42. Song E, Jahng JW, Chong LP, Sung HK, Han M, Luo C, Wu D, Boo S, Hinz B, Cooper MA, et al: Lipocalin-2 induces NLRP3 inflammasome activation via HMGB1 induced TLR4 signaling in heart tissue of mice under pressure overload challenge. Am J Transl Res 9: 2723-2735, 2017 